\title{
Adaptive Mappings for Mouse-Replacement Interfaces
}

\author{
John J. Magee, Samuel Epstein, Eric S. Missimer, and Margrit Betke \\ Department of Computer Science \\ Boston University, Boston, MA, USA \\ $\{$ mageejo, samepst, missimer, betke \}@cs.bu.edu
}

\begin{abstract}
Users of mouse-replacement interfaces may have difficulty conforming to the motion requirements of their interface system. We have observed users with severe motor disabilities who controlled the mouse pointer with a head tracking interface. Our analysis shows that some users may be able to move in some directions easier than other directions. We propose several mouse pointer mappings that adapt to the user's movement abilities. These mappings will take into account the user's motions in two- or three-dimensions to move the mouse pointer in the intended direction.
\end{abstract}

\section{Categories and Subject Descriptors}

H.5.2 [User Interfaces]: Input devices and strategies; H.1.2 [User/Machine Systems]: Human factors

\section{General Terms}

Human Factors

\section{INTRODUCTION}

Mouse-replacement interfaces are used by people with severe motion disabilities that result from disorders such as cerebral palsy, amyotrophic lateral sclerosis, multiple sclerosis or muscular dystrophy. Users' head or facial feature positions are mapped to mouse-pointer coordinates on the screen. Head or facial feature positions may be detected by camera-based systems such as Camera Mouse [1, 2] or SINA [5], or by infrared head-trackers (e.g., [6]). The mapping from the head position in the video frame to the mousepointer position on the screen typically uses a scaling factor. Larger scale factors enable users to move the mouse pointer larger distances with only small head movements.

Our experience with users showed that they were often not able to move their heads in all directions. This resulted in constricted motion of the mouse pointer when scale-based mappings were used. We propose to explore a larger class of mappings that adapt to the user's movement abilites. With an adaptive mapping, the user is able to move the mouse pointer to all positions on the screen with head movements that are most comfortable. In this paper, we first describe our experiences with users of a camera-based mouse-

Copyright is held by the author/owner(s).

This paper was presented at ASSETS'10, October 25-27, 2010, Orlando, Florida, USA.

ACM 978-1-60558-881-0/10/10. replacement system and then introduce adaptive mappings that address the limitations of scale-based mappings.

\section{EXPERIENCE WITH USERS}

Our observations of users with disabilities has shown that they may not be able to comfortably hold their heads vertically. When such users try to move the mouse pointer horizontally, they move it diagonally due to their tilted head orientation.

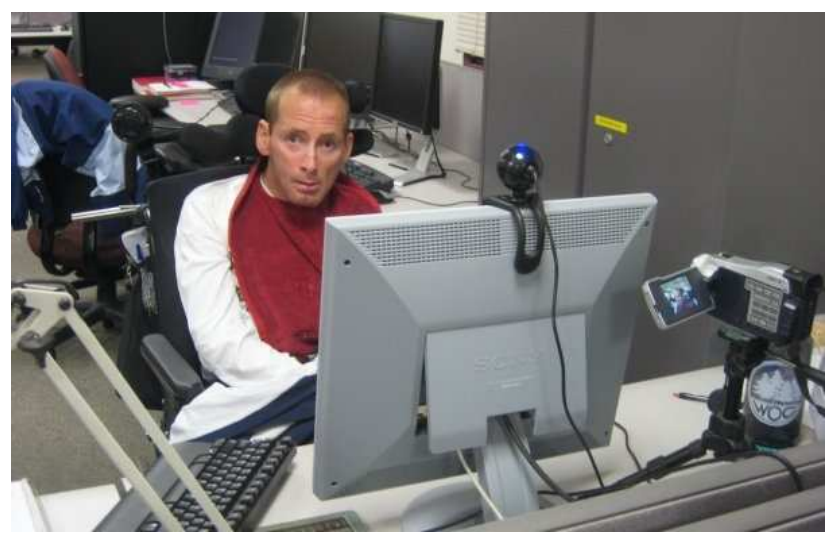

Figure 1: One of the human subjects conducted an experiment where he moved his head left and right in the manner most comfortable to him. His motion was tracked and recorded for analysis.

We observed the difficulties that users had in moving the mouse pointer in certain directions while performing interface experiments. In these tests, circular targets appeared on the screen and the user was asked to move the mouse pointer to the highlighted target. Sometimes the user moved the mouse pointer relatively quickly to the vicinity of the target and then required several attempts to reach the exact target location. Such a trajectory is shown in Figure 2 left. The user had difficulties moving the pointer between horizontal targets. The trajectory in Figure 2 right shows that the digonal movement was much easier for the user to perform.

We also asked an adult subject with cerebral palsy to move his head left and right in a manner that was most comfortable for him (Figure 1). We observed for this particular user that he moved his head upwards when he neared the 

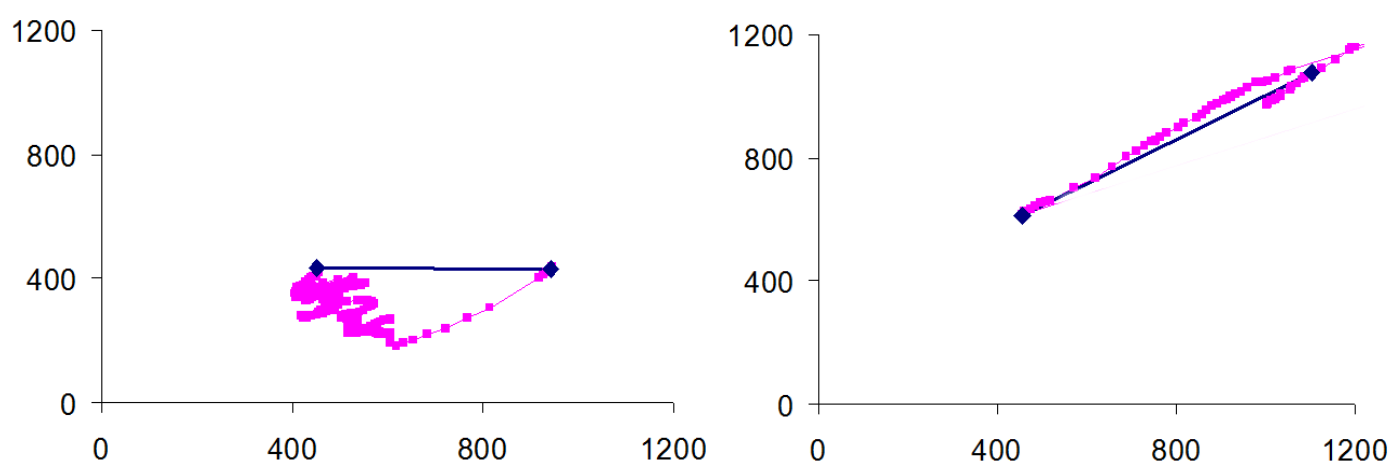

Figure 2: Mouse pointer trajectories in target experiments (desired trajectory blue, performed trajectory pink, units are screen coordinates). Left: The user moves from the right target to the left target. Initially the motion is fast (diagonally downwards) and then requires extensive adjustments to reach the exact target position. Right: The user moves from the left target to the right target with ease.

extremes of his left and right motions. This motion suggests the default linear mouse mapping may not be best suited for this user.

\section{MOUSE MAPPINGS}

We present a framework for modifying the mouse trajectory to determine if "off-axis" (not horizontal or vertical) motion can be compensated for and thus increase the usability of the interface for people who cannot easily move their heads in exactly horizontal or vertical directions. An initial solution to this problem could be to rotate the camera or image to the same angle as the user's head. However, our observations indicate that user motions cannot be compensated with a simple rotation and a more complicated analysis of the intended mouse motion is needed. Based on our observations of users, we propose alternative head-positionto-pointer-coordinate mappings (see Figure 3). In addition

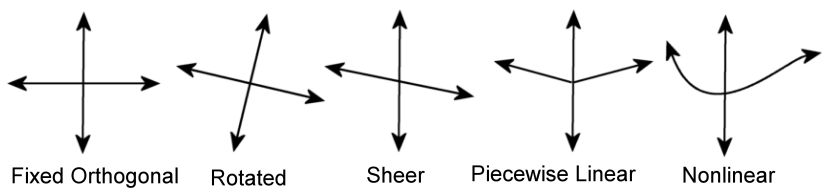

Figure 3: Possible adaptive mappings for mousereplacement systems.

to alternative mappings based on movement in the image plane, we build upon work that explored a multi-camera system that analyzed motions of the user in three dimensions [4]. An experiment was conducted where users of the Camera Mouse system moved the pointer between targets while their motions were recorded with a multi-camera system. A plot of these feature movements is shown in Figure 4. An analysis of feature trajectories shows motions in three dimensions (including towards or away from the camera) that are ignored by the traditional two-dimensional image-plane feature tracker.

Traditional camera-based mouse-replacement systems map the two-dimensional head position $v$ in the video frame into mouse-pointer coordinates $m$. The mapping is typically a

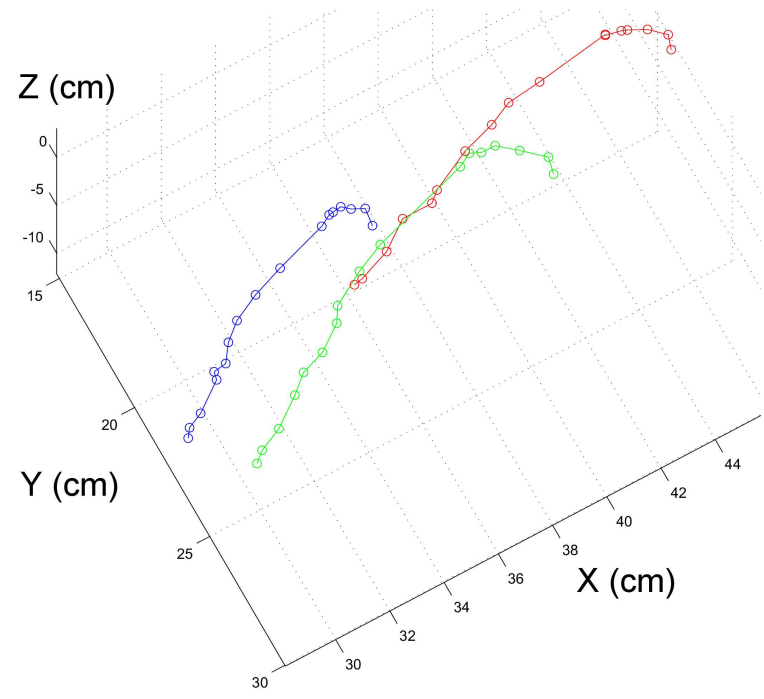

Figure 4: Three-dimensional feature trajectories of a Camera Mouse user performing an experiment.

scaling, $m=k v$, for some scalar $k$. We propose to generalize the mapping to an affine transformation matrix $A$ that allows both rotation and shear along with scaling, such as $m=A v$. We also consider piecewise-linear and non-linear mappings (see Figure 3).

We also examine adaptive mappings for mouse-replacement systems that use more than one camera [4]. Such systems enable stereoscopic reconstruction of the head position $h$ in three-dimensional space (see Figure 4) and can explicitly project $h$ into the two-dimensional mouse-pointer coordinate $m$. (This projection is done implicitly in a one-camera system via the camera projection matrix.) Our future work will incorporate smoothing filters into the mapping [3].

\section{ACKNOWLEDGMENTS}

Funding was provided by the National Science Foundation, HCC grant IIS-0713229. 


\section{REFERENCES}

[1] M. Betke, J. Gips, and P. Fleming. The camera mouse: Visual tracking of body features to provide computer access for people with severe disabilities. IEEE Transactions on Neural Systems and Rehabilitation Engineering, 10(1):1-10, 2002.

[2] Camera Mouse - Innovative software for people with disabilities, http://www. cameramouse.org.

[3] R. Kjeldsen. Improvements in vision-based pointer control. In The 8th International ACM Conference on Computers and Accessibility (ASSETS 2006), Portland, Oregon, USA, pages 189-196, 2006.

[4] J. Magee, Z. Wu, H. Chennamaneni, S. Epstein, D. H. Theriault, and M. Betke. Towards a multi-camera mouse-replacement interface. In 10th International Workshop on Pattern Recognition in Information Systems (PRIS 2010), 2010. 10 pp.

[5] C. Manresa-Yee, J. Varona, F. J. Perales, F. Negre, and J. J. Muntaner. Experiences using a hands-free interface. In The 10th International ACM Conference on Computers and Accessibility (ASSETS 2008), Halifax, Nova Scotia, Canada, pages 261-262, 2008.

[6] SmartNav by Natural Point. http://www . naturalpoint. com/smartnav. 